\title{
Abstracts
}

\section{Physical Activity/Participation Session 2}

Field-testing the Pediatric Neuromuscular Recovery Scale in Children with Spina Bifida

Ardolino, Elizabeth, Flores, Megan, Manella, Kathleen University of St. Augustine for Health Sciences-Austin

Background There is a paucity of reliable and valid outcome measures available for assessing improvements in children with Spina Bifida. The Pediatric Neuromuscular Recovery Scale (Peds NRS) is a valid and reliable 13-item scale that assesses the functional mobility of children with spinal cord injuries (SCI). As children with SCI present with similar impairments to children with Spina Bifida, the Peds NRS may be a viable outcome measure to use in the Spina Bifida population. The purpose of this study was to field test the Peds NRS for practicality of administering the scale in children with Spina Bifida.

Methods One Occupational Therapist and one Physical Therapist completed both online and on-site training on the Peds NRS. The 2 clinicians then administered the Peds NRS on 2 children with Spina Bifida (age 3 years and 6 years). The children's ability to follow the standardized instructions, the ease of scoring the items, and the amount of time to complete the scale were assessed during the field testing.

Results Both children were able to follow the standardized instructions to complete the items on the Peds NRS. The most difficult items to test were In-hand manipulation, Step Retraining and Step Adaptability. Inhand manipulation was challenging to test in the 3 year old due to difficulty with comprehending the task. Step Retraining and Step Adaptability were difficult as neither child had ever walked on a treadmill prior to this testing. Both clinicians were able to score all items without questions, and agreed upon the same score for each child on every item. The amount of time to complete the full scale was 60 min for the 3 year old child, and $70 \mathrm{~min}$ for the 6 year old child.

Conclusions The results of the field testing indicate that the Peds NRS can easily be administered in chil- dren with Spina Bifida. Studies on a larger sample are needed to fully assess the psychometric properties of the scale in this population.

\section{Validity and Reliability of Skill-related Fitness Tests for Wheelchair-using Youth with Spina Bifida Bloemen, Manon ${ }^{1,2}$, Takken, Tim² ${ }^{2}$ Backx, Frank ${ }^{2}$, Vos, Marleen ${ }^{1}$, Kruitwagen, Cas $^{2}$, de Groot, Janke ${ }^{1,2}$ ${ }^{1}$ HU University of Applied Sciences Utrecht \\ ${ }^{2}$ University Medical Center Utrecht}

Background Assessment and optimizing fitness in youth with chronic conditions like Spina Bifida (SB) are important goals in paediatric rehabilitation. Valid and reliable skill-related fitness tests are lacking for wheelchair-using youth with SB. Therefore the objective of this study is to determine validity and reliability of the Muscle Power Sprint Test (MPST), 10x5 Meter Sprint Test (10x5MST), slalom test and one stroke push test (1SPT) in wheelchair-using youth with SB.

Methods Fifty-three wheelchar-using children (519 years) with SB participated. The MPST, 10x5MST, slalom test en 1SPT were assessed. Construct validity of the the MPST was determined by comparing results with the arm-cranking Wingate Anaerobic test (WAnT) using paired t-tests and Pearson Correlation Coefficients, while content validity was assessed using time based criteria for anaerobic testing. Construct validity of the 10x5MST, slalom test and 1SPT was analyzed by hypothesis testing using Pearson Correlation Coefficients and Multiple Regression. For reliability, Intra Class Correlation coefficients (ICC) and smallest detectable changes (SDC) were calculated.

Results For the MPST, mean exercise time of four sprints was $28.1 \mathrm{sec}$. ( $\pm 6.6 \mathrm{sec}$.). Correlations between the MPST and WAnT were high $(r>0.72, p<$ 0.01). Excellent correlations were found between the 10x5MST and slalom test $(r=0.93, p<0.01)$, while 
correlations between the 10x5MST or slalom test and MPST and 1SPT were moderate $(r=-0.56--0.70$; $r=0.56, p<0.01)$. The 1SPT was explained for $38 \%$ by wheelchair mass (Beta -0.489$)$ and total upper muscle strength (Beta 0.420). All ICCs were excellent (ICC > 0.95) but the SDCs varied widely.

Conclusions The MPST, 10x5MST and slalom test are valid and reliable tests in wheelchair-using youth with SB for measuring respectively anaerobic performance or agility. For the 1SPT, both validity and reliability are questionable.

\section{Developmental Wheelchair Mobility Skills in Spinal Defects \\ Wilson, Pamela ${ }^{1,2}$, Blanchard, Alesia ${ }^{1,2}$, Steiner, Julie $^{1,2}$, O’Keeffe, Shannon ${ }^{1,2}$, Palmer, Claire ${ }^{2}$, Scaramuzzi, Gayle ${ }^{1}$, Clayton, Gerald H. ${ }^{1,2}$ \\ ${ }^{1}$ Children's Hospital Colorado \\ ${ }^{2}$ University of Colorado, School of Medicine}

Background There are limited data available on the developmental acquisition of manual wheelchair skills in the young child with a disability. Although an adult wheelchair skills test does exist, less advance skills are not represented. We designed the Pediatric Assessment of Manual Wheelchair Skills (PAMWS) tool to identify developmental milestones acquired over time.

Methods The child is fit with a wheelchair and the PAMWS is based on clinic observation of 39 defined skills that are scored as absent, developing, or proficient (0-2). These skills are further divided into categories based upon their complexity and difficulty. Each skill is observed by 2 personnel (junior and senior) who have been trained to use the tool effectively in the spinal defects and spinal cord injury population. Each subject is then re-examined at subsequent regularly scheduled clinic visits.

Results The median number of visits was 2 (range: 15). Subjects were rated by two clinicians at each visit. The average age at first visit was 30 months (SD: 14) and ranged from 9.1 to 54.7 months. Statistical considerations indicate that the rates of skill acquisition (slope) for each of the levels are different (Basic $>$ Advanced $>$ Pre-Mobility) and each level has a different initial intercept depending on rate of acquisition will become fully competent at different ages. Maximum competency of Pre and Basic skills are achieved at similar times while advance skills mature later. There were no statistically relevant differences between assessors. Conclusions Expertise in the 3 different skill levels is accrued at different rates and early ages exhibit dif- ferent beginning inherent levels. Developmentally, 5 and 11 years of age appear to be important wheelchair motor skill milestones. This preliminary study suggests that the PAMWS tool may be useful in following wheelchair skill development in young children and we suggest there may be some utility for it in identifying atypical skill growth.

Validity of a Wheelchair Perceived Exertion Scale (WHEEL Scale) for Arm Ergometry Exercise in People with Spina Bifida

Crytzer, Theresa M. ${ }^{1,2,3}$, Cheng, Yu-Ting ${ }^{2}$, Robertson, Robert J. ${ }^{4}$, Dicianno, Brad E. ${ }^{1,3,5,6}$

${ }^{1}$ Veterans Affairs Pittsburgh Healthcare System, Human Engineering Research Laboratories

${ }^{2}$ University of Pittsburgh, Department of Rehabilitation Science and Technology

${ }^{3}$ University of Pittsburgh Medical Center, Center for Assistive Technology

${ }^{4}$ University of Pittsburgh, Department of Education, Health and Physical Activity

${ }^{5}$ University of Pittsburgh, School of Medicine Department of Physical Medicine and Rehabilitation

${ }^{6}$ University of Pittsburgh Medical Center, Mercy Hospital, Spina Bifida Clinic

Background Perceived exertion scales are valid, low cost tools that can be used by exercise physiologists and physical therapists to monitor exertion during exercise tests or physical therapy sessions; however, there is a scarcity of validated perceived exertion scales designed specifically for people with disabilities who are wheelchair users. The WHEEL Rating of Perceived Exertion (RPE) scale is a new scale that was adapted for wheelchair users from the OMNI RPE scale. The WHEEL Scale portrays color photos of a person in a wheelchair paired with the verbal descriptors and associated numeric values that were previously validated for children and adolescents without disabilities.

Methods This study assessed the concurrent and construct validity of the Borg 6-20 Scale and WHEEL Scale based on the results of a symptom limited arm ergometry exercise stress test.

Results Twenty-four participants with Spina Bifida were recruited ( $M$ age $=32.1 \mathrm{yr}$., $S D=13.0$, range $=$ 17-71; 12 female; 2 adolescents). Significant, moderate, positive correlations were observed between power output and relative heart rate and power output to relative $\mathrm{VO}_{2}$ peak. A moderate, significant correlation between physiologic criterion variables and the rating of 
perceived exertion derived from the Borg Scale and the WHEEL Scales was found.

Conclusions Concurrent validity was supported by the following findings: (1) relative heart rate was significantly correlated with the Borg (Kendall's $\tau=0.41$ ) and WHEEL Scales ( $\tau=0.44)$, and relative VO2 was significantly correlated with the Borg Scale $(\tau=0.46)$ and WHEEL Scale $(\tau=.47)$; (2) construct validity was supported by the finding that the Borg Scale and the WHEEL Scale shared significant variance $(\tau=$ 0.70). The WHEEL Scale shows strong potential for use in this cohort subsequent to further testing and validation, demonstrating internal consistency.

\section{Personal and Environmental Factors to Consider when Aiming to Improve Physical Activity in Youth with Spina Bifida \\ Bloemen, Manon ${ }^{1,4}$, Verschuren, Olaf ${ }^{2,3}$, van Mechelen, Claudia ${ }^{1}$, Borst, Hanneke ${ }^{1}$, de Leeuw, Arina $^{1}$, van der Hoef, Marsha ${ }^{1}$, de Groot, Janke ${ }^{1,4}$ \\ ${ }^{1}$ HU University of Applied Sciences Utrecht \\ ${ }^{2}$ Brain Center Rudolf Magnus \\ ${ }^{3}$ De Hoogstraat Rehabilitation \\ ${ }^{4}$ Wilhelmina Children's Hospital, University Medical Center Utrecht}

Background Youth with Spina Bifida (SB) are less fit and active than peers. While recent studies have shown benefits of training, the increased fitness does not sustain or lead to increased physical activity (PA). Therefore, it seems important to explore which factors are associated with PA. The objective of this study is to describe personal and environmental factors associated with PA as experienced by these children and the parents, in order to develop interventions to improve PA in youth with SB.

Methods Eleven semi-structured interviews with parents of children with SB aged 4-7 years, nine focus groups with youth with SB ( $n=33$, age $8-18$ years) and eight focus groups with their parents $(n=31)$ were conducted, recorded and transcribed verbatim. Two independent researchers analyzed the data. Central themes for PA were constructed, using the model for PA for Persons with a Disability (PAD model) as a background scheme.

Results Youth with SB encountered personal and environmental factors associated with PA on all levels of the PAD model. Bowel and bladder care, competence in skills, sufficient fitness, medical events and self-efficacy were important personal factors. Environ- mental factors that were associated with PA included the support from other people, the use of assistive devices for mobility and care, adequate information regarding possibilities for adapted sports and accessibility of playgrounds and sports facilities.

Conclusions A variety of personal and environmental factors were either positively or negatively associated with PA. An individual approach, assessing possibilities rather than overcoming barriers may be a good starting point when setting up interventions to improve PA. Therefore, assessment of both personal and environmental factors associated with PA should be standard care within multidisciplinary programs aimed to encourage healthy active lifestyles in youth with SB.

\section{Exercise Prescription using a Group Normalized Rating of Perceived Exertion in Adolescents and Adults with Spina Bifida}

Crytzer, Theresa M. ${ }^{1,2}$, Keramati, Mariam ${ }^{3}$,

Robertson, Robert J. ${ }^{4}$, Cheng, Yu-Ting ${ }^{2}$, Dicianno, Brad E. ${ }^{1,3,5,6}$

${ }^{1}$ Veterans Affairs Pittsburgh Healthcare System, Human Engineering Research Laboratories

${ }^{2}$ University of Pittsburgh, Rehabilitation Science and Technology

${ }^{3}$ University of Pittsburgh, Department of Physical Medicine and Rehabilitation

${ }^{4}$ University of Pittsburgh, School of Education Health and Physical Activity

${ }^{5}$ University of Pittsburgh Medical Center, Center for Assistive Technology

${ }^{6}$ University of Pittsburgh Medical Center Mercy Hospital, Adult Spina Bifida Clinic

Background Exercising at a target rating of perceived exertion (RPE) equivalent to the ventilatory breakpoint $(\mathrm{Vpt})$ provides a training stimulus to improve cardiopulmonary fitness in non-disabled individuals. The perceptual-physiologic link between RPE and Vpt, termed the Group Normalized Perceptual Response, has not yet been determined for people with Spina Bifida who are wheelchair users.

Methods Participants completed a symptom limited arm ergometer stress test. During the test, participants were asked to rate their perceived exertion on the WHEEL Scale and the Borg 6-20 Scale.

Results Of the 29 total subjects, 19 achieved Vpt (Group A) while the remaining 10 did not (Group NA). No significant differences were found between Groups A and NA for gender, age, BMI, history of 
Chiari II malformation, mobility status, Physical Activities Scale for Individuals with Physical Disabilities, or $\mathrm{VO}_{2 \text { peak }}$. However, there was a significantly greater ( $p=0.044)$ number of individuals with restrictive lung disease in Group NA than Group A. The $\% \mathrm{VO}_{2 \text { peak }}$ at Vpt for Group A was $61.76 \pm 16.26$ (range 36.6$\left.99.7 \% \mathrm{VO}_{2 \text { peak }}\right)$. A graded cycle ergometer test indicated that the WHEEL Scale and Borg Scale RPE at Vpt were $5.74 \pm 2.58$ (range 0-10) and $13.95 \pm 3.50$ (range 6-19) respectively. A significant $(p<0.05)$ linear regression model was developed: Borg Scale RPE $=(1.22 \times$ WHEEL Scale RPE $)+7.14$ and used to construct a WHEEL Scale RPE to Borg Scale RPE conversion table, facilitating intermetric prescription of a target RPE.

Conclusions For subjects with Spina Bifida, a Group Normalized WHEEL Scale RPE of $5.74 \pm 2.58$ and a Borg Scale RPE of $13.95 \pm 3.50$ can be used to prescribe and self-regulate arm ergometer exercise intensity equivalent to the Vpt. Pulmonary restriction may be a limitation to high intensity exercise in people with Spina Bifida who do not demonstrate Vpt or achieve peak exercise.

\section{Adapted, Inclusive, Exclusive, or Segregated PE: What is Wrong with this Picture?}

Vanderbom, Kerri A. ${ }^{1}$, Lai, Byron ${ }^{1}$, Taylor, Jennifer ${ }^{2}$

${ }^{1}$ University of Alabama at Birmingham

${ }^{2}$ Western Oregon University

Background Physical Education (PE) in schools is an important place where youth can meet their recommended daily physical activity (PA) and begin to adopt lifelong PA behavior. However, students with disabilities, including students with Spina Bifida, are less likely to be active participants in these settings. Consequently, physical inactivity rates are 4.5 times higher than their non-disabled peers. While there is research on teacher's experiences in inclusive PE, there is little insight to the experiences of students with mobility disabilities (SwMD). The purpose of this session is to discuss the current situation of SwMD in PE from a social justice lens, legislation and rights of SwMD and parents/caregivers, and strategies to improve inclusive $\mathrm{PE}$ in schools.

Methods The presentation will be grounded in the results from a qualitative study that was conducted to gain an understanding of SwMD experiences in PE. Three focus groups were conducted with teens from four different states $(n=14)$. Each lasted one hour and included open-ended questions around their knowledge about PA and health, experiences in PE, and solutions to address barriers. All focus groups were transcribed and coded for major themes and sub-themes.

Results Four major themes identified from the study were: inclusion as a social justice issue, knowledge/ awareness of disability, barriers and facilitators of PE participation, and overcoming barriers in PE/athletics. Conclusions As we know from participatory action research and knowledge translation, to successfully address the issue, SwMD need to be included in the solution-building process. It has been over 40 years since legislation was passed to ensure the inclusion of students with disabilities in PE. The perspectives of SwMD will help academics better prepare future and current teachers, practitioners, and doctors with strategies on inclusion and how to address barriers to participation in PE. 